\title{
Calidad de vida \\ y trasplante renal en mayores de 65 años
}

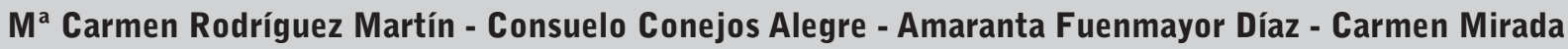

Ariet - Esther Sanz Izquierdo - Carmen Torruella Barraquer - Antonio Vela Ballestero

Departamento de Enfermería. Fundación Puigvert. Barcelona

\section{Resumen}

La calidad de vida se define como "la percepción personal de un individuo de su situación de vida, dentro del contexto cultural y de valores en el que vive, y en relación con sus objetivos, expectativas, valores e intereses".

El objetivo de este trabajo es conocer la calidad de vida de los pacientes trasplantados renales mayores de 65 años. Estudio cualitativo, descriptivo y retrospectivo. La muestra abarcó un total de 31 pacientes mayores de 65 años que fueron trasplantados en nuestro centro desde julio de 2003 hasta julio de 2006. El instrumento de recogida de información fue doble: una encuesta de 11 ítems y el cuestionario SF-36.

El aspecto peor valorado por los encuestados (51,6 puntos) se refería a la capacidad de realizar un esfuerzo físico intenso. Sin embargo, actividades de menor intensidad recibieron una puntuación más elevada (88,7 puntos). Los pacientes mayores de 65 años que han recibido un trasplante renal, perciben una mejoría importante en su calidad de vida respecto al período anterior. Esto se refleja en una ampliación del abanico de actividades a realizar.

\section{Correspondencia: \\ Carmen Rodríguez Martín \\ Servicio de Nefrología \\ Fundación Puigvert \\ C/ Cartagena 340-350 \\ 08025 Barcelona \\ crodriguez@Fundacio-puigvert.es}

\section{PALABRAS CLAVE: \\ - CALIDAD DE VIDA \\ - ANCIANO \\ - TRASPLANTE RENAL \\ - CUESTIONARIO SF-36}

\section{Quality of life and kidney transplants in over-65s}

\section{Abstract}

Quality of life is defined as "the personal perception of an individual of his or her life situation, within the cultural context and the context of the values in which he or she lives, and in relation to his or her goals, expectations, values and interests".

The aim of this study is to determine the quality of life of patients over 65 years of age who have undergone kidney transplants. A qualitative, descriptive and retrospective study. The sample encompassed a total of 31 patients aged over 65 who received kidney transplants at our centre between July 2003 and July 2006. The instrument used to compile the information was in two parts: a survey of 11 items and questionnaire SF-36.

The aspect that received the lowest rating from the patients surveyed (51.6 points) related to the capacity for intense physical efforts. However, activities of lower intensity received a higher score (88.7 points). Patients aged over 65 who have received a kidney transplant perceive an important improvement in their quality of life compared to the period prior to the transplant. This is reflected in an extension of the range of activities they can carry out. 
KEY WORDS:

- QUALITY OF LIFE

- ELDERLY

- KIDNEY TRANSPLANT

- QUESTIONNAIRE SF-36

\section{Introducción}

La calidad de vida se define, según la OMS, como "la percepción personal de un individuo de su situación de vida, dentro del contexto cultural y de valores en el que vive, y en relación con sus objetivos, expectativas, valores e intereses ${ }^{\prime \prime}$. Se trata, por tanto, de un concepto que abarca aspectos objetivos y subjetivos que reflejan el grado de bienestar físico, emocional y social de cada individuo ${ }^{2}$. De este modo, los indicadores de medida de la calidad de vida han ido evolucionando en los últimos años; mientras que a finales de los años 60 primaban los indicadores objetivos relacionados con aspectos materiales cuantificables, a partir de la década de los 90 adquirieron mayor relevancia los indicadores subjetivos individuales.

El concepto de calidad de vida relacionada con la salud (CVRS) incorpora una perspectiva distinta a la puramente biológica, ampliando el foco de atención a parcelas relacionadas con el funcionamiento social, la accesibilidad a los servicios sanitarios o la percepción subjetiva del bienestar general del individuo ${ }^{3}$. Así pues, bajo esta perspectiva, la CVRS representa "las respuestas individuales a los efectos físicos, mentales y sociales que la enfermedad produce sobre la vida diaria, lo cual influye sobre la medida en que se puede conseguir la satisfacción personal en las circunstancias de la vida" ${ }^{\prime 4}$.

Además, la calidad de vida puede referirse no sólo a la pérdida de salud a causa de determinada enfermedad como la insuficiencia renal crónica, sino también a la situación vital que se genera tras haber recibido un tratamiento médico como el trasplante renal. Ambos aspectos están íntimamente relacionados, ya que la percepción de calidad de vida tras el tratamiento tiene mucho que ver con la percibida anteriormente durante el período de enfermedad.

En un estudio realizado sobre calidad de vida en el trasplantado renal, ésta se conceptualizó como "la cualidad que tienen los pacientes de regresar a una vida normal, después de haber sido trasplantados, reincorporándose a actividades familiares, sociales, culturales, laborales, etc., que no podía realizar por las complicaciones de la insuficiencia renal crónica" ${ }^{\prime \prime}$. EI resultado reflejaba la posibilidad de retomar aquellas actividades que los pacientes habían abandonado por su dependencia de algún tipo de tratamiento sustitutivo, mejorando la percepción de calidad de vida en este sentido. Otros estudios sobre la calidad de vida en trasplantados renales reflejan una mejoría en el estado físico y algunos aspectos de la vida diaria como la ampliación de la dieta ${ }^{6}$. Sin embargo, el trasplantado "no es un paciente exento de morbilidad ya que continúa necesitando un control médico especializado y frecuente", además de estar "sometido continuamente a una medicación inmunosupresora no exenta de efectos secundarios y a una serie de complicaciones médicas que son habituales en él", por lo que permanece la característica de enfermo crónico ${ }^{7}$.

La prevalencia de la insuficiencia renal aumenta con la edad, de modo que más del $44,2 \%$ de pacientes con insuficiencia renal crónica terminal son mayores de 60 años $^{8}$. La hemodiálisis es la técnica de depuración extracorpórea más utilizada en estos casos aunque se asocia generalmente a una peor calidad de vida. El trasplante renal se considera una alternativa terapéutica en pacientes mayores de 65 años con insuficiencia renal crónica terminal ya que no existen diferencias significativas en la evolución -tasa de creatinina plasmática, causa de la muerte- en relación a pacientes trasplantados de menor edad.

El objetivo de este trabajo es conocer la calidad de vida de pacientes trasplantados renales mayores de 65 años desde una doble perspectiva: la medición de indicadores estandarizados de salud recogidos en el cuestionario SF-36, así como el análisis de la percepción subjetiva de cada sujeto.

\section{Personas y Método}

Estudio cualitativo, descriptivo y retrospectivo. La muestra abarcó inicialmente un total de 45 pacientes mayores de 65 años que fueron trasplantados en nuestro centro desde julio de 2003 hasta julio de 2006. Los criterios de inclusión fueron: ser mayor de 65 años en el momento del trasplante; haber transcurrido 1 año, como mínimo, desde la realización del 
trasplante; injerto funcionante, y no presentar problemas de comunicación ni de comprensión en el momento de la entrevista. De un total de 45 pacientes, 14 fueron finalmente excluidos del estudio por no cumplir con dichos criterios.

El instrumento de recogida de información fue doble: una encuesta de 11 ítems elaborada específicamente para el estudio, y el cuestionario de calidad de vida SF-36. Ambos fueron respondidos mediante entrevista telefónica durante los meses de septiembre, octubre y noviembre de 2007. La duración de las entrevistas fue de 15 a 20 minutos.

La encuesta fue elaborada con el objetivo de conocer la autopercepción de cada paciente en relación a su estado de salud en el momento de la entrevista. Comprendió un total de 11 ítems distribuidos de la siguiente forma: 6 de tipo objetivo (edad, sexo, edad en el momento del trasplante, tiempo transcurrido desde el trasplante y posibles causas de ingreso en la etapa posterior) y 5 de ámbito subjetivo (puntuación de la calidad de vida percibida antes y después del trasplante, percepción de mejoría, actividades físicas realizadas y repercusiones dietéticas).

El cuestionario SF-36 (Short Form General Survey) abarca un total de 36 ítems que cubren ocho dimensiones del estado de salud, y detecta tanto estados positivos como negativos. Para cada dimensión se recodifican y se suman los ítems, transformándose en una escala del 0 al 100. Las dimensiones evaluadas son: funcionamiento físico, rol físico, dolor, salud general, vitalidad, funcionamiento social, rol emocional y salud mental ${ }^{1}$. Las escalas del SF-36 están ordenadas de forma que a mayor puntuación, mejor es el estado de salud, siendo la peor puntuación el 0 y la mejor el 100.

Para completar la información, se recogieron datos objetivos relativos a la función renal de los pacientes después de ser trasplantados, siendo los principales indicadores la proteinuria y la creatinina plasmática. El análisis estadístico se realizó con el programa SPSS 13.0, y los gráficos con Excel 2003.

\section{Resultados}

\section{Encuesta}

De los 31 pacientes estudiados 14 fueron hombres y 17 mujeres, con una edad media de 71,8 años (rango
66-78 años). La edad media en el momento de la realización del trasplante renal (TR) fue de 69 años (65-76). El tiempo transcurrido desde el TR hasta el momento de la realización del cuestionario fue de una media de 2, 77 años (1-4).

La calidad de vida percibida por la propia persona en una escala de 0 a 10 (siendo 0 la peor puntuación y 10 la mejor puntación) fue de una media de 2,94 en el periodo anterior al TR (0-8) y de 9,13 en la etapa posterior (8-10). Desde la realización del trasplante un total de 24 pacientes manifestaron realizar mayor número de actividades (pasear, viajar, deportes), mientras que 7 consideraron que realizan las mismas actividades. Respecto al tipo de dieta, 23 consideraron que han podido ampliar la dieta mientras que 8 continúan con el mismo tipo de dieta.

De los 31 pacientes encuestados, ingresaron 17 pacientes post-TR, siendo las causas: 5 por neumonía, 1 por fiebre, 2 por empeoramiento de la función renal y 9 por otras causas (diabetes, estenosis ureteral, infección vírica...), (figura 1).

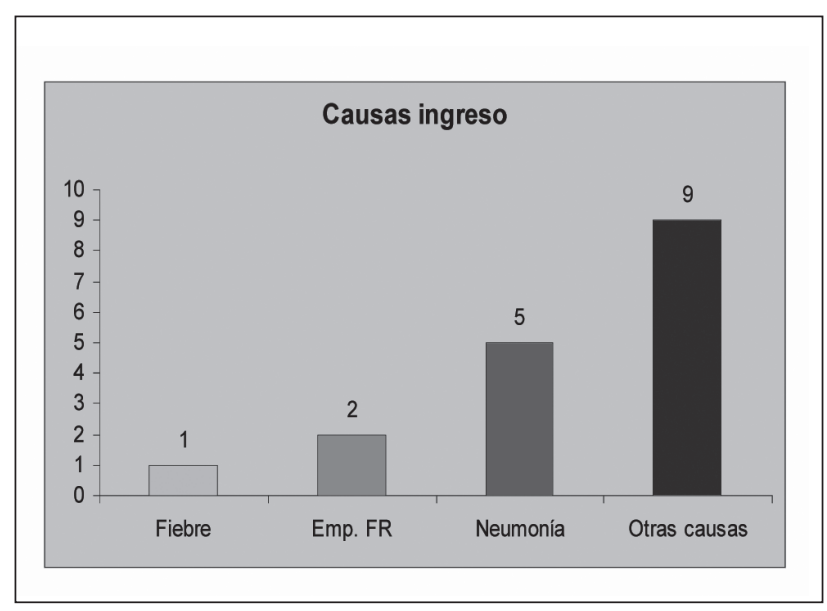

Figura 1.

Respecto a la función renal se obtuvieron unos valores medios, al año del trasplante de: creatinina plasmática $137 \mathrm{mmol}(63 \mathrm{a} 231)$ y proteinuria 0,35 g/24h $(0,07-3,06)$.

\section{Cuestionario SF-36}

En las 8 dimensiones estudiadas se superaron los 60 puntos, siendo la puntuación más baja la referida a la función física (69 puntos) y la más alta a la función social (86 puntos), (tabla 1 ). 


\begin{tabular}{|c|c|c|c|c|}
\hline DIMENSIÓN & VALORACIÓN & $\begin{array}{l}\text { VALOR } \\
\text { MINIMO }\end{array}$ & $\begin{array}{l}\text { VALOR } \\
\text { MÁXIMO }\end{array}$ & $\begin{array}{l}\text { VALOR } \\
\text { MEDIO }\end{array}$ \\
\hline Función física & $\begin{array}{l}\text { Limitación en las actividades } \\
\text { físicas como en el autocuidado } \\
\text { (caminar, bañarse, vestirse, deportes.....) }\end{array}$ & 51,6 & 88,7 & 69 \\
\hline Rol físico & $\begin{array}{l}\text { Grado en que la salud física interfiere } \\
\text { en el trabajo y otras actividades diarias }\end{array}$ & 77,4 & 87 & 82 \\
\hline Dolor corporal & $\begin{array}{l}\text { Intensidad del dolor y su efecto en } \\
\text { las actividades habituales }\end{array}$ & 74,8 & 80,6 & 78 \\
\hline Salud general & Percepción de su estado de salud & 62 & 81,4 & 70,7 \\
\hline Función social & $\begin{array}{l}\text { Grado en que los problemas de salud física } \\
\text { o emocional interfieren en la vida social }\end{array}$ & 84,6 & 87 & 86 \\
\hline Vitalidad & Sentimiento de energía o cansancio & 70,3 & 84,5 & 77 \\
\hline Rol emocional & $\begin{array}{l}\text { Actividades relacionadas con su } \\
\text { estado emocional }\end{array}$ & 77,4 & 77,4 & 77,4 \\
\hline Salud mental & $\begin{array}{l}\text { Sentimiento de angustia, depresión, } \\
\text { felicidad y calma }\end{array}$ & 74,8 & 86,4 & 82 \\
\hline
\end{tabular}

Tabla 1. Resultados por dimensiones

El aspecto peor valorado por los encuestados $(51,6$ puntos) se refería a la capacidad de realizar un esfuerzo físico intenso como correr, levantar objetos pesados o practicar deportes agotadores. Sin embargo, actividades de menor intensidad como caminar una manzana, bañarse 0 vestirse uno mismo recibieron una puntuación más elevada (88,7 puntos).

Por otra parte comparando la percepción de salud actual con la de hace un año, el 36\% manifestó ser mucho mejor, algo mejor el $16 \%$, igual el $42 \%$, algo peor el $3 \%$ y mucho peor el $3 \%$ (figura 2). Respecto a la afirmación "me pongo enfermo más fácilmente que otras personas" la mayoría manifestaron que era falsa.

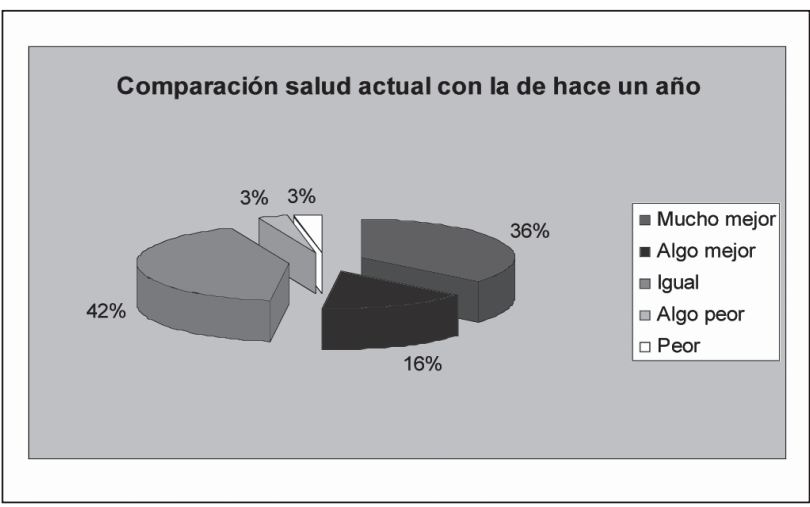

Figura 2.

\section{Discusión}

Los pacientes mayores de 65 años que han recibido un trasplante renal perciben una mejoría importante en su calidad de vida respecto al período anterior ${ }^{1}$. Esto se refleja en una ampliación del abanico de actividades a realizar, como viajar o practicar deporte con moderación, así como en una ampliación de la dieta. La percepción de salud es así mismo mucho mejor que la percibida anteriormente, y no manifiestan la sensación de una mayor vulnerabilidad respecto a la población en general ${ }^{4}$. La dificultad para llevar a cabo un esfuerzo físico intenso está relacionada con la edad avanzada de este colectivo, y posiblemente no esté vinculada al trasplante en sí mismo.

Al año del TR no se observan diferencias significativas en los valores analíticos (creatinina plasmática, proteinuria), respecto a pacientes trasplantados con edad inferior a 65 años, por lo que consideramos que la edad no incide en el resultado del trasplante renal.

Sería interesante en el futuro poder medir la calidad de vida con un instrumento de medida más flexible en las respuestas (entrevistas en profundidad, respuestas abiertas...) que nos permita valorar en profundidad otros matices y percepciones del individuo ${ }^{6}$. 
Estos resultados nos llevan a considerar el trasplante renal en personas de edad avanzada como tratamiento sustitutivo muy válido y que contribuye de forma decisiva a mejorar la calidad de vida objetiva y subjetiva de la persona.

\section{Bibliografía}

1. Ureña A, Fernández 0, Arango A y Rebollo P. Calidad de vida en trasplante renal. En: Libro de comunicaciones del XXI Congreso de la Sociedad Española de Enfermería Nefrológica. Barcelona: HOSPAL; 1996.

2. Aranzabal J, Lavari R, Magaz A, López. Calidad de vida de los trasplantados de órganos. 1992. http:// donación.organos..va.es/info-sanitaria/proceso/calidad.htm

3. López $M^{a}$ A, Rodríguez B, Gómez E, Ortega F. Evaluación de la calidad de vida relacionada con la Salud en enfermos en tratamiento con hemodiálisis periódica incluidos en lista de espera para TR. En: Libro de comunicaciones del XXIV Congreso de la Sociedad Española de Enfermería Nefrológica. Barcelona: HOSPAL; 2004. p. 277-280.
4. Ruiz Mª J, Román M, Martín G, Alférez $M^{a} J$, Prieto D. Calidad de vida relacionada con la salud en las diferentes terapias sustitutivas de la insuficiencia renal crónica. Rev Soc Esp Enferm Nefrol 2003; 6(4): 222-232.

5. Alarcón C, Aguilar 0, Jiménez A, Manrique C. Calidad de vida en pacientes con Trasplante renal medida a través de índice de Karnofsky en un hospital general. Revista Medicina crítica y terapia intensiva. 2002; 119-123.

6. Oliva A, Vivó $M^{\mathrm{a} J}$. Percepción de la calidad de vida en el paciente trasplantado. En: Libro de comunicaciones del XXIV Congreso de la Sociedad Española de Enfermería Nefrológica. Barcelona: HOSPAL; 1999.

7. Junyent $E$, Pujolar N. Calidad de vida en el paciente trasplantado. Revista Biseden $1^{\circ}$ trimestre. 1992. 17-23.

8. Generalitat de Catalunya. Departament de salud. Registre de malats renals de Catalunya. Informe estadístico. 2004.

9. Saudan P, Berney T et al. Trasplante renal en ancianos: pronóstico a largo plazo. 2000. www. Bago.com/ bago/bagooarg/biblio/alerg36web.htm. 